\title{
Mechanisms of the Magneto-Optical Activity of Rare-Earth Ions in Rare-Earth Iron Garnet Single Crystals
}

\begin{abstract}
N.I. Tsidaeva, V.V. Abaeva And T.T. Magkoev
Department of Physics, North-Ossetian State University, 46 Vatutina str., Vladikavkaz, 362025 Russia

The magnetic and magneto-optical properties of the typical representatives of three rare-earth iron garnets (RIG) groups: with heavy rare-earth elements $\mathrm{Yb}, \mathrm{Er}, \mathrm{Dy}$, Tb; with elements from the middle of lanthanide series $\mathrm{Gd}, \mathrm{Sm}$, and with light rare-earth element $\mathrm{Nd}$ are presented. In contradistinction to other work on the Faraday rotation, which were done only at $1152 \mathrm{~nm}\left(8696 \mathrm{~cm}^{-1}\right)$, here we present FR spectra obtained in the energy region $5500-20000 \mathrm{~cm}^{-1}$ with high optical resolution. The investigations have been done at temperatures of $5,82,130$, $295 \mathrm{~K}$ using magnetic field up to $25 \mathrm{kOe}$ applied parallel to the [111] crystallographic axis of the crystals. It has been shown that the contribution proportional to the magnetic field and independent of temperature to the mixing of the ground state multiplets exceeds the paramagnetic contribution in YbIG, ErIG, GdIG, SmIG. In Tb and Dy iron garnets contributions from the two mechanisms have opposite signs, and the paramagnetic mechanism gives the greatest contribution to the Faraday rotation. Nevertheless, the contribution of the diamagnetic mechanism, caused by the influence of the exchange field in the iron sublattices on rare-earth ions, is significant, and it is necessary to take it into account. Anomalously large magneto-optical activity is observed in NdYIG. This is the result of contributions of the same sign and approximately equal in magnitude from the paramagnetic and diamagnetic mechanisms.
\end{abstract}

PACS: $75.50 . \mathrm{Gg}, 78.20 . \mathrm{Ls}$

\section{Introduction}

In recent years, the optical and magneto-optical (MO) properties of ferrimagnetic garnets have attracted increasing interest, from the physical or the application points of view. Dispersive MO effects in pure and substituted rare-earth iron garnets (RIG) have been the subject of numerous experimental and theoretical investigations [1-11].

The origin of the MO activity of the rare-earth ion in RIG is not well understood. Therefore, in this work we investigate the mixed and pure rare-earth garnets, and try to draw some coherent conclusions.

Optical properties of RIG are significantly influenced by two-particle processes that remove selection rules on spin and parity in the visible region, making certain transitions allowed, most likely the forbidden change-transfer transitions. There is, therefore, interest in distinguishing between contributions of allowed and forbidden electric dipole transitions to the MO activity and studying the main non-resonant contribution to the allowed electric dipole transitions, which are located in ultraviolet region. The narrow line widths of the observed intra-configuration $f-f$ transitions of the rare-earth ion allow us defining and excluding (in spectral measurements) the contribution of these transitions to the total rotation observed in the immediate region of the absorption bands only.

We present here for the first time the MO properties of $\mathrm{Yb}_{3} \mathrm{Fe}_{5} \mathrm{O}_{12}, \mathrm{Er}_{x} \mathrm{Y}_{3-x} \mathrm{Fe}_{5} \mathrm{O}_{12} \quad(x=0.4,1.0 ; 1.5$,
$2.5 ; 3.0), \mathrm{Dy}_{3} \mathrm{Fe}_{5} \mathrm{O}_{12}, \mathrm{~Tb}_{3} \mathrm{Fe}_{5} \mathrm{O}_{12}, \mathrm{Gd}_{3} \mathrm{Fe}_{5} \mathrm{O}_{12}, \mathrm{Sm}_{3} \mathrm{Fe}_{5} \mathrm{O}_{12}$, $\mathrm{Nd}_{x} \mathrm{Y}_{3-x} \mathrm{Fe}_{5} \mathrm{O}_{12}(x=0.5,0.96,1.39,1.72)$ single crystals and in particular Faraday rotation (FR) in energy region 5500-20000 $\mathrm{cm}^{-1}$. FR measurements were performed for the same experimental conditions as a function of temperature and magnetic field oriented along the main crystallographic axis [111].

\section{Experimental}

Using the method of flux growth under 10 bar of oxygen pressure, single crystals of $\mathrm{Yb}_{3} \mathrm{Fe}_{5} \mathrm{O}_{12}, \mathrm{Er}_{x} \mathrm{Y}_{3-x} \mathrm{Fe}_{5} \mathrm{O}_{12}$ $(x=0.4,1.0,1.5,2.5,3.0), \mathrm{Dy}_{3} \mathrm{Fe}_{5} \mathrm{O}_{12}, \mathrm{~Tb}_{3} \mathrm{Fe}_{5} \mathrm{O}_{12}$, $\mathrm{Gd}_{3} \mathrm{Fe}_{5} \mathrm{O}_{12}, \mathrm{Sm}_{3} \mathrm{Fe}_{5} \mathrm{O}_{12}$ were synthesized. X-ray diffraction measurements were in agreement with the garnet Ia $3 d$ structure. Polished plates $-20-250 \mu \mathrm{m}$ thick oriented perpendicular to the [111] and [100] axes were obtained from the same "as grown" crystal to avoid the slight Y concentration difference which can occur between different crystals of the same batch. NdYIG samples were grown from the $\mathrm{PbO}: \mathrm{PbFe}_{2}: \mathrm{B}_{2} \mathrm{O}_{3}$ flux melts by spontaneous nucleation. The compositions of the samples were determined from the published relationship between the compositions and the lattice constant. Polished plates (30-35 $\mu \mathrm{m}$ thickness) were oriented perpendicular to [111] axes.

FR measurements $\mathrm{F}$ were performed at temperatures 5 , $82,130,295 \mathrm{~K}$ under a magnetic field of up to $25 \mathrm{kOe}$ applied parallel to the [111] direction. In contradistinction to other researches of the FR, which were done only at 
$1152 \mathrm{~nm}\left(8696 \mathrm{~cm}^{-1}\right)[2,6]$, we present the FR spectra obtained in energy region $5500-20000 \mathrm{~cm}^{-1}$ with a high optical resolution $0.12 \mathrm{~cm}^{-1}$ using a modulation technique. The experimental accuracy is estimated to $\pm 2 \%$ for $\Phi$. It is noted that the samples are cooled at the lowest temperature in the absence of a magnetic field prior to FR measurements. The rotation angle was measured with $0.1^{\circ}$ accuracy.

Field dependences of the FR and the differential FR susceptibility $\mathrm{d} \Phi / \mathrm{d} H$ were measured using the compensation method (without the modulation of magnetic field) at two wavelengths $1152 \mathrm{~nm}\left(8696 \mathrm{~cm}^{-1}\right)$ and $1064 \mathrm{~nm}$ $\left(9400 \mathrm{~cm}^{-1}\right)$ (for comparison with other researches). The wavelengths used in this study are in the transparency window of all garnets.

The crystallographic direction [111] is the easy magnetization axes at three studied temperatures $(82,130$, and $295 \mathrm{~K}$ ) (above the compensation point). The direction of light propagation and the direction of applied magnetic field coincide with the [111] direction. In this geometry of an experiment, effects of magnetic birefringence are eliminated and crystals are uniaxial.

\section{Results and discussion}

Absorption spectra of three samples of $\operatorname{Er}_{x} \mathrm{Y}_{3-x} \mathrm{Fe}_{5} \mathrm{O}_{12}$ $(x=0.4,1.5,2.5)$, for example, are plotted in Fig. 1 [9].

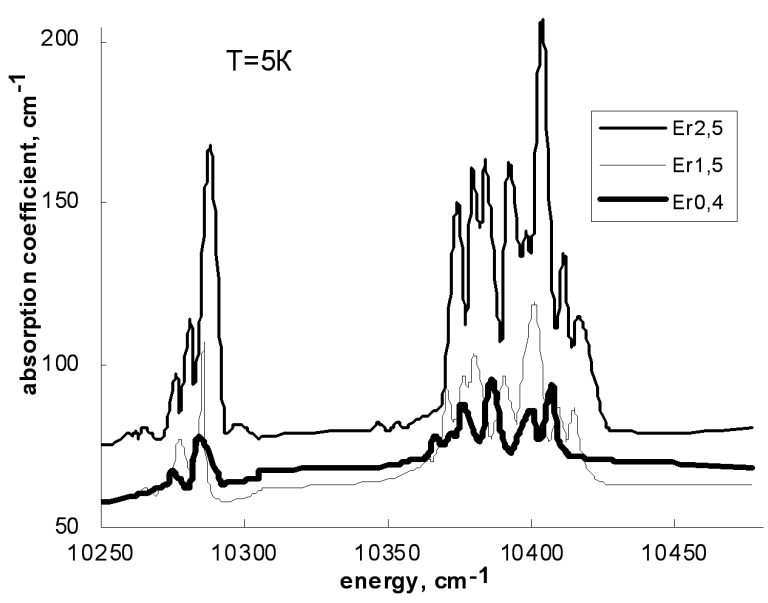

Fig. 1. Absorption spectra of three samples of $\mathrm{Er}_{x} \mathrm{Y}_{3-x} \mathrm{Fe}_{5} \mathrm{O}_{12}(x=0.4,1.5,2.5)$.

There are two absorption maxima in all the absorption spectra. The first maximum in the energy region $10500 \mathrm{~cm}^{-1}$ is identified with an ${ }^{6} A_{1 \mathrm{~g}}\left({ }^{6} S\right)-{ }^{4} T_{1 \mathrm{~g}}\left({ }^{4} G\right)$ transition in $\mathrm{Fe}^{3+}$ ions occupying octahedral sites in the garnet structure, the second maximum in the energy region $16000 \mathrm{~cm}^{-1}$ is identified with ${ }^{6} A_{1}\left({ }^{6} S\right)-{ }^{4} T_{1}\left({ }^{4} G\right)$ transition in $\mathrm{Fe}^{3+}$ ions occupying tetrahedral sites.

As we said in Introduction, to study these transitions in detail was not our aim: for our purposes we only have determined the appropriate energy region. In our calculation we excluded those regions of the absorption spectra corresponding to absorption lines of the rare-earth.
The FR dispersion in all the studied samples is characterized by a monatonic rise with frequency of the FR due to the contribution of allowed electric transitions in $\mathrm{Fe}^{3+}$ and $\mathrm{R}^{3+}$ ions (or associated molecular complexes) whose frequencies are in the ultraviolet region. Those spectra are shown in Fig. 2 [5].

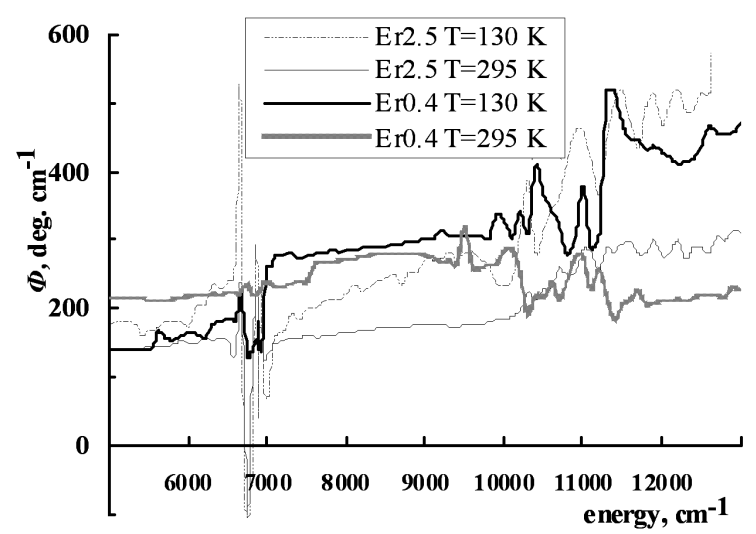

Fig. 2. Frequency dependences of Faraday rotation of $\mathrm{Er}_{x} \mathrm{Y}_{3-x} \mathrm{Fe}_{5} \mathrm{O}_{12}(x=0.4,2,5)$.

The peculiarities of the FR involving narrow intraconfigurational $f-f$ absorption bands of $\mathrm{R}^{3+}$ ion and a wider $d-d$ band ${ }^{6} A_{1 \mathrm{~g}}\left({ }^{6} S\right){ }^{4} T_{1 \mathrm{~g}}\left({ }^{4} G\right)\left(\approx 10900 \mathrm{~cm}^{-1}\right)$ of octahedral $\mathrm{FeO}_{6}^{9-}$ complexes can be seen against the background of the monotonous rise of $\mathrm{FR}$.

The results of our experiments may be explained via the quantum theory of FR, taking into account the influence of effective magnetic fields on the splitting of the ground and excited multiplets of rare-earth ions and the mixing of wave functions of ground and excited multiplets of rare-earth ions. The electric dipole transition between the $4 f^{3}$ and $4 f^{2} 5 d$ configurations may be studied by taking into account the spin-orbit crystal field and superexchange interaction in the development [7].

The Faraday rotations induced by the electric (el) and magnetic (mag) dipole transitions are proportional to the magnetic moments $\left(M_{a}, M_{d}, M_{\mathrm{c}}\right)$ of the different magnetic ions $[2,8]$ :

$$
\begin{array}{r}
(\mathrm{RIG})=F_{\mathrm{el}}^{+} F_{\mathrm{mag}}= \pm\left(A_{e}+A_{m}\right)\left|M_{a}\right| \\
\quad \mp\left(D_{e}+D_{m}\right)\left|M_{d}\right| \pm\left(C_{e}+C_{m}\right)\left|M_{c}\right|,
\end{array}
$$

where the upper sign is valid for $T<T_{\text {comp }}$, the lower for $T>T_{\text {comp }}$.

The MO activity of $\mathrm{R}^{3+}$ ions was analyzed using the conventional assumption that contributions to the total $\mathrm{FR}$ of $\mathrm{R}^{3+}$ and iron sublattices are independent of each other. The $\mathrm{R}^{3+}$-sublattice contribution to the FR was determined as the difference of the FR of the studied crystal and the FR of a pure YIG. A deviation of concentration dependences from linear was observed for some of the $f-f$ absorption bands. The FR depends linearly on the concentration of $\mathrm{Er}^{3+}$ and $\mathrm{Nd}^{3+}$ ions as we deduced from the absorption bands. 
Let us consider the influence of allowed electric dipole transitions. They are the principal contribution to the MO activity, for the influence of forbidden $f-f$ and $d-d$ transitions is exhibited only in the immediate region of absorption bands and can be eliminated if calculations are performed only for those spectral regions where the contribution of forbidden transitions can be neglected.

The FR is usually treated in magnetically ordered crystals as proportional to sublattice magnetisation [6]. The isolation of the non-resonant contribution within this model will also result in a severe dependence of the MO constant of $\mathrm{Er}^{3+}$ ions on the temperature as in [6]. Thus let us consider the FR taking into account that the representation of the temperature independent contribution directly depended on the exchange interaction.

The $\mathrm{R}^{3+}$-sublattice contribution to the FR at high temperatures can be considered to be the contribution of free ions and if the diamagnetic contribution is neglected, it can be expressed as

$$
F_{\mathrm{RE}}=C_{m} M_{R}+C_{e} M_{R}+D H_{\mathrm{eff}},
$$

where $M_{R}$ is the magnetic moment of the $\mathrm{R}^{3+}$ ion in crystal, $C_{m} M_{R}$ is the gyromagnetic $\mathrm{FR}$ and $C_{e}, D-\mathrm{MO}$ constants of the gyroelectric FR. The paramagnetic contribution $C_{e} M_{R}$ is determined by a thermal population of energy levels of the ground manifold of the $\mathrm{R}^{3+}$ ion and the contribution of the mixing mechanism $D H_{\text {eff }}$ arises from an admixture of wave functions of excited manifolds into the wave functions of the ground manifold by the exchange interaction. The effective field is related to the external magnetic field $H$ and molecular field $H_{m}$ by the expression

$$
H_{\text {eff }}=H-g(g-1)^{-1} H_{m} .
$$

Here the $g$-factor is taken to be equal to the Landé factor of the free ion $(g=1.2)$. The contribution of the magnetic dipole transitions was calculated from the relation $C_{m}=1440 n e g x / m c^{2} a^{3}$, where $n=2.2$ is the mean value of the refractive index and $a$ is the parameter of the elementary cell. The MO constants were determined from the observed FR by the simultaneous solution of Eq. (2) for two temperatures. In our calculations we used data for magnetization of $\mathrm{R}^{3+}$-sublattice and for molecular fields $H_{m}(295 \mathrm{~K})$ and for magnetization of iron sublattices (known from magnetic and MO studies) which were believed to be equal to sublattice magnetizations in YIG. The molecular field acting on the $\mathrm{R}^{3+}$-sublattice was assumed to be proportional to the magnetization of the iron sublattice.

MO constants $C_{e}$ grow in magnitude with increasing frequency and are well approximated by a characteristic expression for paramagnetic contribution dependence

$$
C_{e}(\lambda)=C_{e}^{0}\left(\lambda^{2}-\lambda_{0}^{2}\right)^{-1},
$$

where $\lambda$ is the wavelength (Fig. 3). The wavelength of the effective resonance absorption for ErIG $\lambda_{0}=150 \mathrm{~nm}$, for DyIG $\lambda_{0}=325 \mathrm{~nm}$ and for TbIG $\lambda_{0}=238 \mathrm{~nm}$. The proximity of this value to the wavelength of the first allowed transitions of the $\mathrm{Er}^{3+}$ ion shows that the gyroelectric FR is likely be determined by the direct interconfigurational transitions of the $\mathrm{Er}^{3+}$ ions. The wavelength of effective resonance absorption for Nd-YIG is $\lambda_{0}=510 \mathrm{~nm}$.

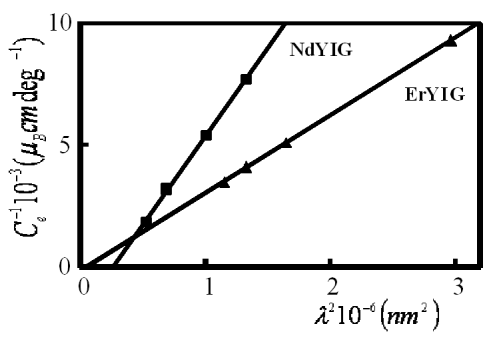

Fig. 3. The wavelength dependences of MO constant $C_{e}$ of Er-YIG and Nd-YIG.

The constant $D$ was independently determined using Eq. (2) from the variation of the FR in the external magnetic field in the region of technical saturation.

When analyzing the values of paramagnetic and diamagnetic contributions for some wavelengths we conclude that the absolute values of diamagnetic contributions to gyroelectrical FR in YbIG, ErIG, SmIG and GdIG are larger than the paramagnetic contributions; the diamagnetic contribution in YbIG is higher than paramagnetic; in GdIG diamagnetic contribution is ten times more than paramagnetic; and in SmIG gyroelectrical FR of $\mathrm{R}^{3+}$ -sublattice is practically completely diamagnetic.

For SmIG we consider the values of the tabulated results as estimated (therefore they are given in parentheses). As the $\mathrm{Sm}^{3+}$ magnetic moment is small, the determined quantities can change substantially, depending on the chosen values of the $\mathrm{R}^{3+}$-sublattice magnetization. However, this does not change the basic conclusion concerning the dominant role of diamagnetic contribution $D H_{\text {eff }}$ to FR.

Similar changes of spectra FR of YbIG and Nd-YIG to the relation of $\mathrm{Y}_{3} \mathrm{Fe}_{5} \mathrm{O}_{12}$ are explained on the basis of two different mechanisms.

The magnetooptical coefficient $C_{e}$ of $\mathrm{Yb}_{3} \mathrm{Fe}_{5} \mathrm{O}_{12}$ is positive in contrast to the corresponding coefficients of other RIG. It makes positive the paramagnetic contribution in FR $\mathrm{R}^{3+}$-sublattice; this results in experimentally observable curves, if one takes into account that the magnetic moment of $\mathrm{R}^{3+}$-sublattice in this case is directed against the resulting magnetic moment of two Fe-sublattices at $T=295 \mathrm{~K}$ and $T=82 \mathrm{~K}$.

There is a somewhat different situation in case of $\mathrm{Nd}$ YIG. The magnetic moment of the $\mathrm{Nd}^{3+}$ ion is parallel to the resulting magnetic moment of the Fe-sublattices, in common with all light elements with less than half closed - $f$ shell atom in the RIG structure. This is the reason why the paramagnetic contribution to FR has a negative value. When the contribution is summed with equal and, in some cases with exceeded in value negative in sign diamagnetic contribution, it results in large negative experimentally observed values of $\mathrm{FR}$ in $\mathrm{Nd}^{3+}$ system. 
The contribution of the mixing mechanism $D H_{\text {eff }}$ exceeds in magnitude the paramagnetic contribution $C_{e} M_{R}$ and is of the opposite sign in Er-YIG. The value of the paramagnetic constant $C_{e}$ appeared to be close to values of this parameter in paramagnetic crystals ErAIG and ErGaG, and yet the values of the $D$ constant in paramagnetic garnets are practically negligible.

Anomalous great magneto-optical activity is the result of joint action of similar in sign and approximately equal in quantity paramagnetic and diamagnetic mechanisms in $\mathrm{Nd}-\mathrm{Y}$ garnets system, but not the only paramagnetic as authors [7] declare.

\section{Conclusion}

Measurements of frequency dependence of $\mathrm{FR}$ in pure $\mathrm{Yb}_{3} \mathrm{Fe}_{5} \mathrm{O}_{12}, \mathrm{Dy}_{3} \mathrm{Fe}_{5} \mathrm{O}_{12}, \mathrm{~Tb}_{3} \mathrm{Fe}_{5} \mathrm{O}_{12}, \mathrm{Gd}_{3} \mathrm{Fe}_{5} \mathrm{O}_{12}$, $\mathrm{Sm}_{3} \mathrm{Fe}_{5} \mathrm{O}_{12}$, Er-YIG and Nd-YIG substituted garnets have allowed us to deduce the dominant role of the mechanism of wave-function mixing in FR in these materials:

- the diamagnetic mechanism dominates the FR in $\mathrm{Yb}, \mathrm{Er}, \mathrm{Sm}, \mathrm{Gd}$ garnets;

- in $\mathrm{Tb}$ and Dy iron garnets contributions of paramagnetic and diamagnetic mechanisms have opposite signs, and the paramagnetic mechanism gives the greatest contribution in FR. Nevertheless the contribution of the diamagnetic mechanism, caused by the influence of the exchange field of the iron sublattices on $\mathrm{R}^{3+}$-ions, is significant, and it is necessary to take this into account;

- it has been shown that the contribution of the mixing of the ground state multiplets proportional to magnetic field and independent of temperature exceeds the paramagnetic contribution to FR in $\mathrm{Y}$ -substituted erbium iron garnets Er-YIG. The concentration dependence of the MO coefficients justifies the single-ion nature of MO activity of ErYIG [11];
- the temperature independent diamagnetic mechanism plays an important role in formation of FR in Y-substituted neodymium iron garnets. Anomalously large MO activity is the result of joint action of contributions of the same sign and approximately equal magnitude of paramagnetic and diamagnetic mechanisms in Nd-YIG.

\section{Acknowledgments}

This work was supported by Ministry of Education and Science of Russian Federation within the subject plan and under the analytical departmental target program grant N 2.1.1/3938.

\section{References}

[1] W. Wang, Y. Yue, G. Liu, J. Alloys Comp. 509, 1489 (2011).

[2] W.A. Crossley, R.W. Cooper, J.L. Page, R.P. Van Stapele, Phys. Rev. 181, 896 (1969).

[3] P.B.A. Fechine, F.M.M. Pereira, M.R.P. Santos, F.P. Filho, A.S. de Menezes, R.S. de Oliveira, J.C. Góes, L.P. Cardoso, A.S.B. Sombra, J. Phys. Chem. Solids 70, 804 (2009).

[4] W. Wang, G. Liu, J. Appl. Phys. 104, 013914 (2008).

[5] N. Tsidaeva, V. Abaeva, J Alloys Comp. 418, 145 (2006).

[6] N. Adachi, T. Hibi, T. Okuda, J. Magn. Magn. Mater. 177-181, 233 (1998).

[7] F. Zhang, Y. Xu, J. Yang, M. Guillot, J. Phys., Condens. Matter. 12, 7287 (2000).

[8] W. Zhao, Sens. Actuat. A, Phys. 89, 250 (2001).

[9] N.I. Tsidaeva, J. Alloys Comp. 374, 160 (2004).

[10] W. Wang, J. Appl. Phys. 102, 063905 (2007).

[11] R.L. Streever, J. Magn. Magn. Mater. 241, 137 (2002). 